\title{
Discussion: Triangular labyrinth side weirs with one and two cycles
}

Seyed Mahmood Borghei PhD

Professor, Department of Civil Engineering, Sharif University of Technology, Tehran, Iran

\section{Mohammad Ali Nekooie MSc}

PhD Candidate, Faculty of Civil Engineering, Universiti Teknologi Malaysia (UTM), Skudai, Malaysia

\section{Hadi Sadeghian MSC}

PhD Candidate, School of Civil Engineering, University of Tehran, Tehran, Iran

\section{Mohammad Reza Jalili Ghazizadeh PhD}

Assistant Professor, Department of Water Engineering, Abbaspour University of Technology, Tehran, Iran

\section{Ali Parvaneh MSc}

Former Graduate Student, Department of Civil Engineering, Sharif University of Technology, Tehran, Iran

\section{James Yang PhD}

Professor, Department of Civil and Architectural Engineering, Royal Institute of Technology (KTH), Stockholm, Sweden; Vattenfall R\&D, Älvkarleby, Sweden

Amir Javaheri MSC

PhD Candidate, School of Civil and Construction Engineering, Oregon State University, Corvallis, OR, USA

Abdorreza Kabiri-Samani PhD

Associate Professor, Department of Civil Engineering, Isfahan University of Technology, Isfahan, Iran

\section{NOTATION}

$F_{1} \quad$ Froude number at upstream end of side weir

$L \quad$ width of side weir

$L_{\mathrm{ef}} \quad$ total weir crest length of labyrinth side weir

$p \quad$ height of weir crest

$Q_{1} \quad$ total discharge in main channel upstream of the side weir

\section{Contribution by A. Parvaneh, J. Yang,}

\section{A. Javaheri and A. Kabiri-Samani}

The contributors would like to thank the authors (Borghei et al., 2013) for their investigation into the hydraulic characteristics of triangular labyrinth side weirs with one and two cycles. The contributors, however, would like to add a few points about triangular labyrinth side weirs in comparison with trapezoidal and asymmetric labyrinth side weirs.

According to the literature, the length of trapezoidal, triangular and asymmetric labyrinth side weirs is computed considering Equations 35-37, respectively (Figure 20).

35. $L_{\mathrm{ef}}=4 a+2 b$

36. $L_{\mathrm{ef}}=\frac{L}{\sin \alpha}$

37. $L_{\mathrm{ef}}=\frac{L}{\sin \alpha}+\frac{L}{\tan \alpha}$

In accordance with Emiroglu et al. (2014) and the authors' work, the length of triangular labyrinth side weirs and their corresponding trapezoidal and asymmetric labyrinth side weirs are presented in Tables 4 and 5 .

Therefore, the length of triangular labyrinth side weirs are, respectively, $16-25 \%$ greater than those of trapezoidal labyrinth side weirs, and $34-87 \%$ less than asymmetric labyrinth side weirs (Tables 6 and 7).

Based on the data presented by Emiroglu et al. (2014) and their equation for trapezoidal labyrinth side weirs, the authors claimed that although the crest length of triangular labyrinth side weirs is $16-25 \%$ greater than that of trapezoidal side weirs, the discharge coefficient of trapezoidal labyrinth side weirs is far greater than that of triangular labyrinth side weirs (Table 8).

To perform a better comparison of triangular labyrinth, trapezoidal labyrinth, asymmetric labyrinth and rectangular side weirs, part of the experimental data by Borghei and Parvaneh (2011) and Parvaneh et al. (2012) is presented in Table 9.

As illustrated in Figure 21, the discharge coefficient of trapezoidal labyrinth side weirs (presented by Emiroglu et al. (2014)) is far greater than that of triangular labyrinth side weirs (investigated in the original paper by Borghei et al. (2013)) despite a $16 \%$ shorter crest length. They also declared in their previous contributions that the discharge coefficient of triangular and trapezoidal labyrinth side weirs with one cycle is, respectively, $1 \cdot 5-4 \cdot 5$ and $1 \cdot 5-5 \cdot 0$ times greater than that of 


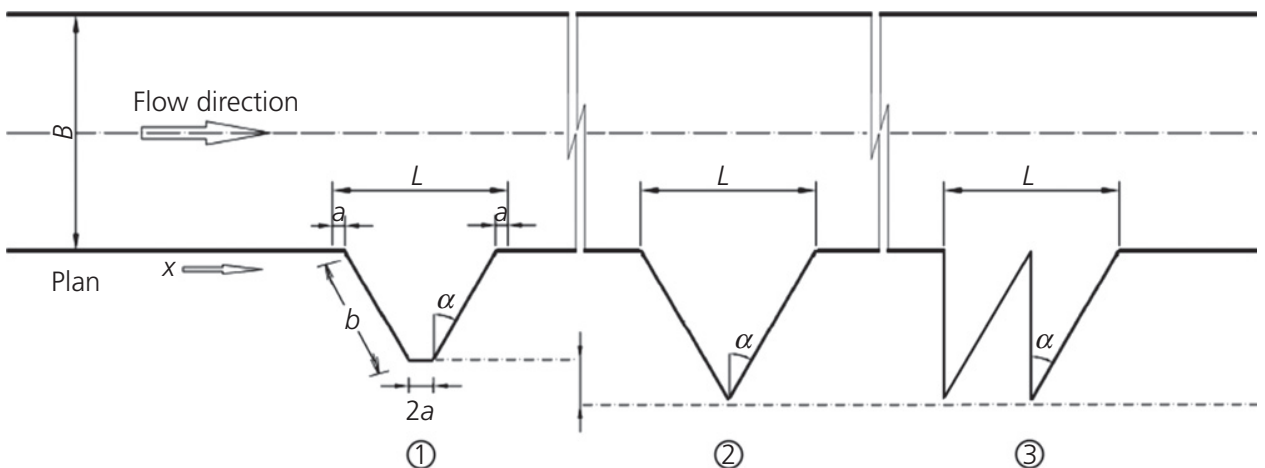

Figure 20. Comparative plan view of trapezoidal (1), triangular (2), and asymmetric (3) labyrinth side weirs with one cycle

\begin{tabular}{|c|c|c|c|c|c|c|c|c|c|}
\hline \multirow{2}{*}{$\begin{array}{l}\text { Labyrinth side weir with } \\
\text { one or two cycles }\end{array}$} & \multicolumn{3}{|c|}{$L=25 \mathrm{~cm}$} & \multicolumn{3}{|c|}{$L=50 \mathrm{~cm}$} & \multicolumn{3}{|c|}{$L=75 \mathrm{~cm}$} \\
\hline & $\alpha=30 \cdot 0^{\circ}$ & $\alpha=22 \cdot 5^{\circ}$ & $\alpha=16 \cdot 0^{\circ}$ & $\alpha=30.0^{\circ}$ & $\alpha=22 \cdot 5^{\circ}$ & $\alpha=16 \cdot 0^{\circ}$ & $\alpha=30 \cdot 0^{\circ}$ & $\alpha=22.5^{\circ}$ & $\alpha=16 \cdot 0^{\circ}$ \\
\hline Trapezoidal & 43 & 54 & 72 & 86 & 108 & 145 & 129 & 162 & 217 \\
\hline Triangular & 50 & 65 & 91 & 100 & 131 & 181 & 150 & 196 & 272 \\
\hline
\end{tabular}

Table 4. The crest length of triangular and trapezoidal labyrinth side weirs with the geometry of Emiroglu et al. (2014)

\begin{tabular}{|c|c|c|c|c|c|c|c|c|c|c|c|c|}
\hline \multirow{2}{*}{$\begin{array}{l}\text { Labyrinth side weir } \\
\text { with one or two cycles }\end{array}$} & \multicolumn{4}{|c|}{$L=30 \mathrm{~cm}$} & \multicolumn{4}{|c|}{$L=40 \mathrm{~cm}$} & \multicolumn{4}{|c|}{$L=60 \mathrm{~cm}$} \\
\hline & $\alpha=70^{\circ}$ & $\alpha=60^{\circ}$ & $\alpha=45^{\circ}$ & $\alpha=30^{\circ}$ & $\alpha=70^{\circ}$ & $\alpha=60^{\circ}$ & $\alpha=45^{\circ}$ & $\alpha=30^{\circ}$ & $\alpha=70^{\circ}$ & $\alpha=60^{\circ}$ & $\alpha=45^{\circ}$ & $\alpha=30^{\circ}$ \\
\hline Trapezoidal & 32 & 35 & 42 & 60 & 43 & 46 & 57 & 80 & 64 & 69 & 85 & 120 \\
\hline Triangular & 43 & 52 & 72 & 112 & 57 & 69 & 97 & 149 & 86 & 104 & 145 & 223 \\
\hline
\end{tabular}

Table 5. The crest length of triangular and asymmetric labyrinth side weirs with the geometry of the authors' work

\begin{tabular}{lccc}
\hline & \multicolumn{3}{c}{$L=25,50,75 \mathrm{~cm}$} \\
\cline { 2 - 4 } Side weir & $\alpha=30 \cdot 0^{\circ}$ & $\alpha=22 \cdot 5^{\circ}$ & $\alpha=16 \cdot 0^{\circ}$ \\
\hline Trapezoidal labyrinth & $16 \%$ & $20 \%$ & $25 \%$
\end{tabular}

Table 6. Increase in the crest length of triangular labyrinth side weirs compared to the trapezoidal labyrinth side weirs with the geometry of Emiroglu et al. (2014)

rectangular side weirs (Emiroglu and Kaya, 2011; Emiroglu et al., 2010). Contrary to their declarations, such a significant increase in the discharge coefficient of weirs with a maximum crest length of 2.6 and 2.9 times that of rectangular side weirs seems abnormal (Table 10).

\begin{tabular}{lcccc}
\hline & \multicolumn{4}{c}{$L=30,40,60 \mathrm{~cm}$} \\
\cline { 2 - 4 } Side weir & $\alpha=70^{\circ}$ & $\alpha=60^{\circ}$ & $\alpha=45^{\circ}$ & $\alpha=30^{\circ}$ \\
\hline Asymmetric labyrinth & $34 \%$ & $49 \%$ & $71 \%$ & $87 \%$ \\
Table 7. Decrease in the crest length of triangular labyrinth side \\
weirs compared to the asymmetric labyrinth side weirs with the \\
geometry of the authors' work
\end{tabular}

Unlike the very high values assigned by Emiroglu et al. (2010) to the discharge coefficient of triangular labyrinth side weirs, the authors have presented more reasonable values of discharge coefficient for the geometries studied. The final results of the current research show that the discharge coefficient of a 


\begin{tabular}{|c|c|c|c|c|c|c|c|c|c|}
\hline \multirow[b]{2}{*}{ Side weir with two cycles } & \multicolumn{3}{|c|}{$L=25 \mathrm{~cm}$} & \multicolumn{3}{|c|}{$L=50 \mathrm{~cm}$} & \multicolumn{3}{|c|}{$L=75 \mathrm{~cm}$} \\
\hline & $\alpha=30 \cdot 0^{\circ}$ & $\alpha=22 \cdot 5^{\circ}$ & $\alpha=16 \cdot 0^{\circ}$ & $\alpha=30.0^{\circ}$ & $\alpha=22 \cdot 5^{\circ}$ & $\alpha=16 \cdot 0^{\circ}$ & $\alpha=30 \cdot 0^{\circ}$ & $\alpha=22.5^{\circ}$ & $\alpha=16.0^{\circ}$ \\
\hline & 1.84 & 1.92 & & $2 \cdot 05$ & $2 \cdot 37$ & - & $2 \cdot 17$ & $2 \cdot 47$ & $2 \cdot 65$ \\
\hline Trapezoidal labyrinth & 4.29 & $4 \cdot 85$ & 4.44 & $4 \cdot 14$ & $4 \cdot 50$ & - & 3.48 & $4 \cdot 12$ & $5 \cdot 31$ \\
\hline
\end{tabular}

Table 8. Maximum values of the discharge coefficient of triangular and trapezoidal labyrinth side weirs to the rectangular side weir (considering the data presented by Emiroglu et al. (2014))

\begin{tabular}{cccccc}
\hline$L: \mathrm{cm}$ & $p: \mathrm{cm}$ & $\alpha: \operatorname{deg}$ & $F_{1}$ & $Q_{1}: 1 / \mathrm{s}$ & Run no. \\
\hline 30 & $7 \cdot 5,10,15$ & 30 & $0.20-0.91$ & $19 \cdot 1-30 \cdot 0$ & 15
\end{tabular}

Table 9. Range of variables studied by Borghei and Parvaneh (2011) and Parvaneh et al. (2012), used in the present discussion

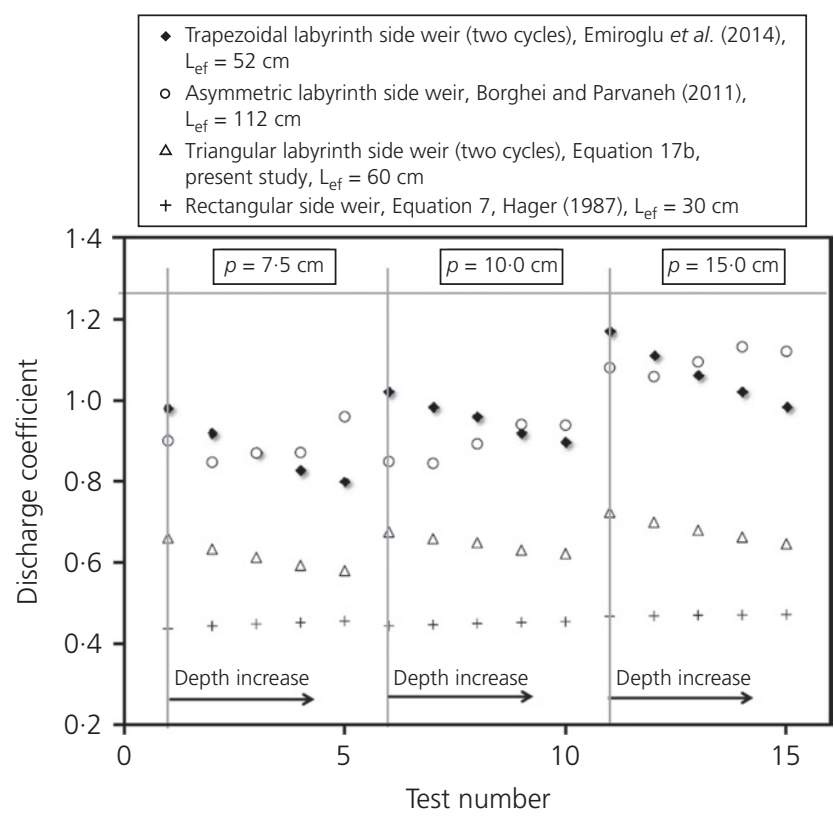

Figure 21. The discharge coefficient of asymmetric labyrinth, trapezoidal labyrinth, triangular labyrinth and rectangular side weirs ( $B=40 \mathrm{~cm}, L=30 \mathrm{~cm}, \alpha=30^{\circ}$ )

triangular labyrinth side weir could rise to more than two times that of a conventional side weir with the same flow and geometric conditions. These results are more consistent for triangular labyrinth side weirs, for which the maximum crest length is $2 \cdot 0$ to $2 \cdot 6$ times larger than that of conventional side weirs (Table 10).

For a given geometry, increasing the flow depth significantly decreases the discharge coefficient of triangular labyrinth side weirs (Parvaneh et al., 2011). According to Figure 21, the

\begin{tabular}{|c|c|c|c|}
\hline \multirow{2}{*}{$\begin{array}{l}\text { Side weir with one } \\
\text { or two cycles }\end{array}$} & \multicolumn{3}{|c|}{$L=25,50,75 \mathrm{~cm}$} \\
\hline & $\alpha=30 \cdot 0^{\circ}$ & $\alpha=22 \cdot 5^{\circ}$ & $\alpha=16 \cdot 0^{\circ}$ \\
\hline $\begin{array}{l}\text { Triangular labyrinth } \\
\text { (Emiroglu et al., 2010) }\end{array}$ & 0.2 & 2.6 & - \\
\hline $\begin{array}{l}\text { Trapezoidal labyrinth } \\
\text { (Emiroglu and Kaya, 2011) }\end{array}$ & $1 \cdot 7$ & $2 \cdot 1$ & 2.9 \\
\hline \multicolumn{4}{|c|}{$\begin{array}{l}\text { Table 10. Ratios of the crest length of triangular and trapezoidal } \\
\text { labyrinth side weirs to the crest length of the corresponding } \\
\text { rectangular side weirs }\end{array}$} \\
\hline
\end{tabular}

discharge coefficient of trapezoidal labyrinth side weirs decreases considerably by increasing the flow depth. A similar conclusion can be offered for trapezoidal weirs.

\section{Authors' reply}

The authors thank the contributors for their interest in their paper. Their clarification of some other aspects of the authors' original study is greatly appreciated.

\section{REFERENCES}

Borghei SM and Parvaneh A (2011) Discharge characteristics of a modified oblique side weir in subcritical flow. Flow Measurement and Instrumentation 22(5): 370-376.

Borghei SM, Nekooie MA, Sadeghian H and Ghazizadeh MRJ (2013) Triangular labyrinth side weirs with one and two cycles. Proceedings of the Institution of Civil Engineers Water Management 166(1): 27-42, http://dx.doi.org/ 10.1680/wama.11.00032.

Emiroglu ME and Kaya N (2011) Discharge coefficient for trapezoidal labyrinth side weir in subcritical flow. Water Resources Management 25(3): 1037-1058.

Emiroglu ME, Kaya N and Agaccioglu H (2010) Discharge capacity of labyrinth side weir located on a straight channel. Journal of Irrigation and Drainage Engineering ASCE 136(1): 37-46.

Emiroglu ME, Aydin MC and Kaya N (2014) Discharge characteristics of a trapezoidal labyrinth side weir with one 
and two cycles in subcritical flow. Journal of Irrigation and Drainage Engineering ASCE 140(5): 1-13.

Hager WH (1987) Lateral out flow over side weirs. Journal of Hydraulic Engineering ASCE 113(4): 491-503.

Parvaneh A, Borghei SM and Jalili Ghazizadeh MR (2011)

Discussion of discharge capacity of labyrinth side weir located on a straight channel. Journal of Irrigation and Drainage Engineering ASCE 137(11): 743-745.

Parvaneh A, Borghei SM and Jalili Ghazizadeh MR (2012)

Hydraulic performance of asymmetric labyrinth side weirs located on a straight channel. Journal of Irrigation and Drainage Engineering ASCE 138(8): 766-772. 